\title{
How many times do I need to see to believe? The impact of intolerance of uncertainty and exposure experience on safety-learning and retention in young adults
}

Article

Accepted Version

Creative Commons: Attribution-Noncommercial-No Derivative Works 4.0

Morriss, J., Wake, S., Lindner, M., McSorley, E. and Dodd, H. (2020) How many times do I need to see to believe? The impact of intolerance of uncertainty and exposure experience on safety-learning and retention in young adults. International Journal of Psychophysiology, 153. pp. 8-17. ISSN 0167-8760 doi: https://doi.org/10.1016/j.ijpsycho.2020.04.012 Available at https://centaur.reading.ac.uk/90720/

It is advisable to refer to the publisher's version if you intend to cite from the work. See Guidance on citing.

Published version at: http://dx.doi.org/10.1016/j.ijpsycho.2020.04.012

To link to this article DOI: http://dx.doi.org/10.1016/j.ijpsycho.2020.04.012

Publisher: Elsevier

All outputs in CentAUR are protected by Intellectual Property Rights law, including copyright law. Copyright and IPR is retained by the creators or other copyright holders. Terms and conditions for use of this material are defined in the End User Agreement. 


\section{www.reading.ac.uk/centaur}

\section{CentAUR}

Central Archive at the University of Reading

Reading's research outputs online 
How many times do I need to see to believe? The impact of intolerance of uncertainty and exposure experience on safety-learning and retention in

\section{young adults}

Jayne Morriss*, Shannon Wake, Michael Lindner, Eugene McSorley \& Helen Dodd

Centre for Integrative Neuroscience and Neurodynamics

School of Psychology and Clinical Language Sciences

University of Reading

Reading

UK

${ }^{*}$ Correspondence:

Jayne Morriss

Centre for Integrative Neuroscience and Neurodynamics

School of Psychology and Clinical Language Sciences

University of Reading

Earley Gate, Whiteknights Campus

RG6 6AH Reading

United Kingdom

j.e.morriss@reading.ac.uk 


\begin{abstract}
Individuals who score high in self-reported Intolerance of Uncertainty (IU) display difficulties updating threat associations to safe associations. Here we sought to determine whether individuals who score high in IU can learn and retain new safety associations if given more exposure. We recorded skin conductance response, pupil dilation and expectancy ratings during an associative threat learning task with acquisition, same-day extinction and next-day extinction phases. Participants $(n=$ 144) were assigned to either a regular exposure (32 trials of same-day and next-day extinction) or extended exposure condition (48 trials of same-day and next-day extinction). We failed to replicate previous work showing that IU is associated with poorer safety-learning indexed via SCR. We found preliminary evidence for promoted safety-retention in individuals with higher Inhibitory IU in the extended exposure condition, relative to individuals with higher Inhibitory IU in the regular exposure condition, indexed via SCR. These findings further our current understanding of the role of IU in safety-learning and -retention, informing models of IU and exposure-based treatments.
\end{abstract}

Keywords: Threat Acquisition, Safety-Learning, Safety-Retention, Intolerance of Uncertainty, Skin Conductance, Pupil Dilation 


\section{Introduction}

The ability to learn and update threat and safety associations is crucial for maintaining health and wellbeing (Milad \& Quirk, 2012; Shin \& Liberzon, 2009). Learning threat associations is adaptive and protects us from potentially dangerous situations. However, when a cue ceases to signal threat, it is adaptive to update this association. Failure to do so can result in dysfunctional fears that affect quality of life. Changes in contingency, such as threat to safety, may not always be obvious; it may take a few experiences to recognise that something that once signalled threat may now signal safety. Uncertainty about changes in contingency from threat to safety in the environment may prolong the learning and retention of new safety associations (Bouton, 2002).

Uncertainty has been identified as an important facet of anxiety and stress disorders (Carleton, 2016a, 2016b; Dugas, Buhr, \& Ladouceur, 2004; Grupe \& Nitschke, 2013). Yet, only recently has the role of individual differences in Intolerance of Uncertainty (IU) (Freeston, Rhéaume, Letarte, Dugas, \& Ladouceur, 1994), a tendency to find uncertainty aversive, been examined in relation to safetylearning (Morriss, Christakou, \& Van Reekum, 2015, 2016) and safety-retention (Dunsmoor, Campese, Ceceli, LeDoux, \& Phelps, 2015; Lucas, Luck, \& Lipp, 2018). More specifically, previous work has shown that higher IU is associated with reduced safety-learning, indexed by greater skin conductance responding and pupil dilation to cues that no longer signal threat during the late part of same-day extinction (i.e. last 8 trials) (Morriss, 2019; Morriss et al., 2015; Morriss \& van Reekum, 2019). Furthermore, high IU is associated with poorer safety-retention, as higher IU individuals show larger skin conductance responding to cues that no longer signal 
threat: (1) during next-day extinction (Dunsmoor et al., 2015), and (2) during sameday extinction after reinstatement (Lucas et al., 2018).

Despite these advancements, it is unclear whether individuals who score high in IU have a fundamental difficulty with safety-learning and safety-retention or whether they simply require more exposure than individuals low in IU i.e. extended extinction sessions across a number of days. Given that IU is transdiagnostic (Gentes \& Ruscio, 2011; McEvoy \& Mahoney, 2012) and that current exposure therapies are based on associative learning principles (Craske, Treanor, Conway, Zbozinek, \& Vervliet, 2014), examining the impact of IU upon exposure experience may reveal crucial information relevant to anxiety and stress disorders. In particular, examining IU in relation to safety-retention across multiple extinction sessions is highly relevant due to high rates of relapse in anxiety and stress disorders (Bandelow, Michaelis, \& Wedekind, 2017). We can speculate that IU may be one of the reasons why after treatment some patients with anxiety disorders relapse i.e. individuals with higher IU have difficulty retaining safety information. Therefore, examining the circumstances under which safety-learning and safety-retention can be promoted in individuals with high IU may facilitate new avenues for clinical research on the role of IU and exposure-based treatments for anxiety and stress disorders (Craske et al., 2014; Knowles \& Olatunji, 2018)

Here we used an associative threat learning task in a relatively large sample $(n=144)$, to assess the relationship between self-reported IU and exposure experience on safety-learning and safety-retention. We measured skin conductance responses, pupil dilation and expectancy ratings whilst participants underwent threat acquisition, same-day extinction and next-day extinction phases. We used an aversive sound as an unconditioned stimulus and visual shape stimuli as conditioned 
stimuli, similar to previous conditioning research including our own (Morriss et al., 2015; Morriss, Christakou, et al., 2016; Neumann \& Waters, 2006). We used a 50\% reinforcement rate during acquisition to sustain conditioning during extinction (Grady, Bowen, Hyde, Totsch, \& Knight, 2016; Leonard, 1975). Participants were assigned to either a regular exposure (32 trials of same-day and next-day extinction) or extended exposure condition (48 trials of same-day and next-day extinction). We matched individuals based on self-reported IU to ensure we had an equal balance of IU in each condition.

In the regular exposure condition, participants underwent same-day and nextday extinction with 32 trials each, similar to the number of trials used in previous research on same-day extinction (Dunsmoor et al., 2015; Lucas et al., 2018; Morriss et al., 2015; Morriss, Christakou, et al., 2016). In the extended exposure condition, participants underwent same-day and next-day extinction with 48 trials each, in line with longer extinction sessions used in prior studies i.e 48-60 trials (Rabinak et al., 2014; Sehlmeyer et al., 2011; Wicking et al., 2016). It has been shown that it is the number of trials not the cumulated duration of trials in extinction that determines safety-learning success (Golkar, Bellander, \& Öhman, 2013). Therefore, differences between the regular and extended exposure conditions should be determined by the difference in the number of trials only. Notably, on next-day extinction, the conditioned response fades more quickly due to re-extinction processes (also known as the extinction retention index), therefore less trials are typically used (Lonsdorf, Merz, \& Fullana, 2019). However, we included more trials in order to compare whether safety-learning is improved for individuals high in IU during next-day extinction, compared to same-day extinction. 
We hypothesised that during threat acquisition, we would observe conditioned responding, indexed by greater skin conductance responding, pupil dilation and expectancy ratings to the learned threat $\left(\mathrm{CS}_{+}\right)$versus safety $(\mathrm{CS}-)$ cues. The first aim was to replicate previous findings regarding safety-learning, safety-retention and IU. Based on previous research, we predicted that in the regular exposure condition higher IU would be associated with a larger conditioned response to the CS+ vS. CScues during the late part of same-day extinction (i.e. last 8 CS+/CS- trials) (Morriss, 2019; Morriss et al., 2015; Morriss, Christakou, et al., 2016; Morriss \& van Reekum, 2019) and the early part of next-day extinction (first 8 CS+/CS- trials) (Dunsmoor et al., 2015).

The proposed study aimed to extend prior findings by examining whether more exposure promotes safety-learning and retention in individuals with higher levels of IU. The first way we attempted to do this was by including more trials during next-day extinction than in previous research. The second way we attempted to do this was by evaluating whether more exposure promotes safety-learning and retention in individuals with higher levels of IU is by comparing conditions that vary in the number of exposure trials across both same-day and next-day extinction. Specifically, we evaluated the hypothesis that individuals with higher IU would show reduced conditioned responding to the CS+ vs. CS- cues in the extended condition relative to the regular condition during: 1 . the late part of same-day extinction (i.e. last 8 CS+/CS- trials); 2. the early and the late parts of next-day extinction. Lastly, we hypothesised that low IU individuals would extinguish similarly regardless of the number of exposure trials on same-day and next-day extinction. These hypotheses were tentative given the lack of research that has examined the impact of more exposure and IU on safety-learning and retention during next-day extinction. 
In line with our previous work (for discussion see Morriss, Christakou \& van Reekum, 2016) we tested the specificity of IU effects by controlling for trait anxiety, assessed by the State-Trait Inventory for Cognitive and Somatic Anxiety (STICSA: Ree, French, MacLeod, \& Locke, 2008). We selected the STICSA because it is considered a purer measure of anxiety, compared to other trait anxiety measures which also feature depressive symptomology (Grös, Antony, Simms, \& McCabe, 2007).

The experimental protocol and hypotheses were preregistered with the Open Science Framework (https://osf.io/2ugpv/).

\section{Method}

\section{Participants}

144 participants were recruited from the University of Reading and local area through the use of advertisements and word of mouth (Sex: 86 female, 58 male; $M$ age $=23.99$ years, $S D=4.42$ years, range $=18-35$ years; Ethnicity: 92 White, 29 Asian, 4 Middle Eastern/Arab, 2 Black, 2 Mixed, and 15 not specified; Sexual Orientation: 104 Heterosexual, 20 Sexual Minorites (lesbian/gay/ bisexual/pansexual), 20 not specified). 6 participants did not return for the second day of testing and for 2 participants there were technical errors, leaving 136 participants with day 1 and day 2 data, and 142 participants with day 1 data.

Participants were recruited if they were between 18-35 years of age. This age range was selected on the basis that there may be additional differences in safetylearning and retention due to age and hormone levels in populations that are under or over this range (Lonsdorf \& Merz, 2017). No other exclusion criteria were used for recruitment. We did not restrict recruitment according to IU score. Participants were 
paid $£ 15$ in total to remunerate them for their time. Participants received $£ 5$ at the end of the first day of testing and $£ 10$ at the end of the second day of testing. The procedure was given ethical approval by the University of Reading Research Ethics Committee.

The experimental data were analysed using multilevel models (MLM), where IU and STICSA scores were entered as a continuous predictor variables. MLMs are more powerful than repeated measures ANCOVA's as they can account for missing cases (Quené \& Van den Bergh, 2004). Despite this, there is no agreed upon method for calculating power and estimating sample size for MLM because of the complexity of the approach (Peugh, 2010; Snijders, 2005). For this reason, appropriate sample sizes were estimated based upon power analyses using and ANCOVA.

The initial power analyses estimated 136 participants. However, we realised that it included an incorrect $f$ value. Therefore, we updated the power analyses to the following. The sample size of this study was based on a power analysis using the average effect size $\left(n^{2} p=.16\right)$ taken from Stimulus $\times$ Time $\times$ IU interactions for SCR magnitude from five previous experiments (4/5 with significant effects of IU)(Morriss, Christakou, \& Van Reekum, 2015, 2016; Morriss \& van Reekum, 2019). The following parameters were used: effect size $f=0.43$ (converted from $n^{2} p=0.16$ ), $\alpha$ error probability $=0.05$, Power $(1-$ error probability $)=0.95$, number of groups $=2$ (regular, extended), numerator $d f=1$, number of covariates $=2$ (IU, STICSA). The total sample size required was $n=73$. Based on the updated power analysis, we oversampled.

\section{Procedure}


On the first day of the experiment, participants were informed about the experimental procedures. Participants were seated in the testing booth and asked to complete a consent form and a set of questionnaires on the computer (see below). One of the researchers assigned participants to either condition (regular, extended) based on IU score, to ensure an even distribution of IU in each condition (Morriss \& van Reekum, 2018). The other researcher who was responsible for testing the participant was blind to condition allocation.

Participants were asked to wash their hands in water without any soap and remove any eye-makeup. Next, physiological sensors were attached to the participants' non-dominant hand and the eyetracker was mounted upon the participants' head. The conditioning task (see "Conditioning task" below for details) was presented on a computer, whilst skin conductance, pupil dilation and behavioural ratings were recorded. Participants were instructed to: (1) maintain attention to the task by looking at the coloured squares and listening to the sounds,

(2) respond to the expectancy rating scales that follow the end of each block of trials, using number keys on the keyboard with their dominant hand and (3) to stay as still as possible.

On the second day (24 hours later), participants received the same instructions as in the first day. The same computer and physiological setup was used as above. Each testing session took approximately 30 minutes in total.

\section{Conditioning task}

The conditioning task was designed using E-Prime 2.0 software (Psychology Software Tools Ltd, Pittsburgh, PA). Visual stimuli were presented at a $75 \mathrm{~Hz}$ refresh rate on a 21 inch colour monitor (DiamondPro, Sony). Participants sat approximately 
$60 \mathrm{~cm}$ from the screen. Visual stimuli were blue and yellow squares with visual angles of $6.16^{\circ} \times 9.07^{\circ}$. The aversive sound stimulus was presented through headphones. The sound consisted of a scream used in our previous experiments (Morriss, Saldarini, \& Van Reekum, 2019; Morriss \& van Reekum, 2019). The volume of the sound was standardized across participants by using fixed volume settings on the presentation computer and verified by an audiometer prior to each session $(90 \mathrm{~dB})$.

The task comprised of three learning phases: threat acquisition, same-day extinction (SDE) and next-day extinction (NDE). In acquisition, one of the coloured squares (blue or yellow) was paired with the aversive $90 \mathrm{~dB}$ sound $50 \%$ of the time (CS+), whilst the other square (yellow or blue) was presented alone (CS-).

Conditioning contingencies were counterbalanced, with half of participants receiving the blue square paired with the US and the other half of participants receiving the yellow square paired with the US. We used a $50 \%$ pairing rate to maximize the unpredictability of the CS+ / US contingency. During the extinction phases, both the blue and yellow squares were presented in the absence of the US. Participants did not receive contingency instructions about any of the phases. Furthermore, there was no break between the acquisition and extinction phases.

The acquisition phase consisted of 24 trials (6 CS+ paired, $6 \mathrm{CS}+$ unpaired, 12 CS-). The regular exposure extinction phases consisted of 32 trials each (16 CS+ unpaired, 16 CS-) and the extended exposure extinction phases consisted of 48 trials each (24 CS+ unpaired, 24 CS-) (see Figure 1). In the regular and extended exposure extinction phases, early is defined as the first 8 CS+/CS- trials, and late is defined as the last 8 CS+/CS- trials. 
Experimental trials were pseudo-randomised such that the first trial of acquisition was always paired and then after all trial types were randomly presented. The coloured squares were presented for a total of $4000 \mathrm{~ms}$. The aversive sound lasted for 1000 ms, which subsided with the offset of the reinforced CS+'s.

Subsequently, a blank screen was presented for 6000 - 8800 ms (Morriss, Saldarini, Chapman, Pollard, \& van Reekum, 2018; Morriss \& van Reekum, 2018).

Blocks of trials in acquisition consisted of 12 trials and in extinction consisted of 16 trials. At the end of each block, participants were asked to rate how much they expected the blue square and yellow square to be followed by the sound stimulus, where the scale ranged from 1 (“Don't Expect”) to 9 (“Do Expect”).

Two other 9-point Likert scales were presented at the end of the experiment on the first day. Participants were asked to rate: (1) the valence and (2) arousal of the sound stimulus. The scales ranged from 1 (Valence: very negative; Arousal: calm) to 9 (Valence: very positive; Arousal: excited).

\section{Questionnaires}

To assess IU and trait anxiety, we administered the Intolerance of Uncertainty Scale (Freeston et al., 1994) and STICSA questionnaires (Ree, French, MacLeod, \& Locke, 2008). The IU measure consisted of 27 items that are rated on a 5-point Likert scale. The STICSA consisted of 21 items that are rated on a 4-point Likert scale.

\section{Rating scoring}

Rating data were reduced for each participant by calculating their average responses for each experimental condition (Acquisition CS+; Acquisition CS-; SDE CS+ Early; 
SDE CS- Early; SDE CS+ Late; SDE CS- Late; NDE CS+ Early; NDE CS- Early; NDE CS+ Late; NDE CS- Late) using the E-Data Aid tool in E-Prime (Psychology Software Tools Ltd, Pittsburgh, PA).

\section{Skin conductance acquisition and scoring}

Physiological recordings were obtained using AD Instruments (AD Instruments Ltd, Chalgrove, Oxfordshire) hardware and software. Electrodermal activity was measured with dry MLT116F silver/silver chloride bipolar finger electrodes that were attached to the distal phalanges of the index and middle fingers of the left hand. A low constant-voltage $\mathrm{AC}$ excitation of $22 \mathrm{mV}_{\mathrm{rms}}$ at $75 \mathrm{~Hz}$ was passed through the electrodes, which were connected to a ML116 GSR Amp, and converted to DC before being digitized and stored. An ML138 Bio Amp connected to an ML870 PowerLab Unit Model 8/30 amplified the skin conductance signal, which was digitized through a 16-bit A/D converter at $1000 \mathrm{~Hz}$. The electrodermal signal was converted from volts to microSiemens using $A D$ Instruments software $(A D$ Instruments Ltd, Chalgrove, Oxfordshire).

Skin conductance responses were marked using ADinstruments software (AD Instruments Ltd, Chalgrove, Oxfordshire) and extracted using Matlab R2017a software (The MathWorks, Inc., Natick, Massachusetts, United States). CS+ unpaired and CS- trials were included in the analysis, but CS+ paired trials were discarded to avoid sound confounds. Skin conductance responses (SCR) were scored when there is an increase of skin conductance level exceeding 0.03 microSiemens (Dawson, Schell, \& Filion, 2000). The amplitude of each response was scored as the difference between the onset and the maximum deflection prior to the signal flattening out or decreasing. SCR onsets and respective peaks were 
counted if the SCR onset was within $0.5-3.5$ seconds (CS response) following CS onset (Morriss, Chapman, Tomlinson, \& Van Reekum, 2018). Trials with no discernible SCRs were scored as zero. SCR magnitudes were square root transformed to reduce skew and z-scored (across conditions and phases) withinsubject to control for interindividual differences in skin conductance responsiveness (Ben-Shakhar, 1985). SCR magnitudes were calculated by averaging SCRtransformed values for each condition (Acquisition CS+; Acquisition CS-; SDE CS+ Early; SDE CS- Early; SDE CS+ Late; SDE CS- Late; NDE CS+ Early; NDE CSEarly; NDE CS+ Late; NDE CS- Late). We defined non-responders as those who responded to $10 \%$ or less of the CS+ unpaired and CS- trials (Morriss, Chapman, et al., 2018; Xia, Dymond, Lloyd, \& Vervliet, 2017). Non-responders were excluded from the SCR analyses.

\section{Pupil dilation acquisition and scoring}

Eye movements were recorded using an Eyelink II eye-tracker with a sampling rate of $250 \mathrm{~Hz}$ (SR Research). Head movements were constrained with a chin-rest at a viewing distance of $60 \mathrm{~cm}$. The eyetracker was calibrated using a standard 3 point grid at the start of the experiment.

Pupil dilation was extracted using Matlab R2017a software (The MathWorks, Inc., Natick, Massachusetts, United States). CS+ unpaired and CS- trials were included in the analysis, but CS+ paired trials were discarded to avoid sound confounds. Blink and saccade artifacts were identified using the associated Eyelink II markers and removed from the pupil dilation data. Pupil dilation was averaged for each 1000 ms window following CS onset, resulting in four windows of $1000 \mathrm{~ms}$ each. These data were baseline corrected by subtracting 1000 ms preceding each 
CS onset from a blank screen (Lonsdorf et al., 2017). Following this pupil dilation data were z-scored (across conditions and phases) within-subject to control for interindividual differences in pupil dilation size (Leuchs, Schneider, \& Spoormaker, 2019). Trials were averaged per stimulus type, time and second window for each participant resulting in the following conditions (Acquisition CS+; Acquisition CS-; SDE CS+ Early; SDE CS- Early; SDE CS+ Late; SDE CS- Late; NDE CS+ Early; NDE CS- Early; NDE CS+ Late; NDE CS- Late).

\section{Ratings, SCR magnitude and pupil dilation analysis}

The analysis was conducted using the mixed procedure in SPSS 25.0 (SPSS, Inc; Chicago, Illinois). We conducted separate MLMs expectancy ratings, SCR magnitude and pupil dilation during acquisition, SDE and NDE. For expectancy ratings and SCR magnitude during the acquisition phase we entered Condition (Regular Exposure, Extended Exposure) and Stimulus (CS+, CS-) at level 1 and individual subjects at level 2. For expectancy ratings and SCR magnitude during SDE and NDE we entered Condition (Regular Exposure, Extended Exposure), Stimulus (CS+, CS-) and Time (Early: first 8 CS+/CS- trials, Late: last 8 CS+/CStrials) at level 1 and individual subjects at level 2. For pupil dilation an additional factor of Second $(1,2,3,4)$ was included in the MLMs.

We included the following individual difference predictor variables into all of the multilevel models: IU and STICSA. In all models, we used a diagonal covariance matrix for level 1. Random effects include a random intercept for each individual subject, where a variance components covariance structure was used. Fixed effects include Condition, Stimulus, Time and Second. We used a maximum likelihood estimator for the multilevel models and corrected post-hoc tests for multiple 
comparisons using the Benjamini-Hochberg False Discovery Rate procedure (Benjamini \& Hochberg, 1995). Acceptable values for multiple comparisons had to be below the following: Acquisition rating, $p<.016$; Acquisition SCR, $p<.016$; Acquisition pupil dilation, $p<.007$; SDE rating, $p<.021$; SDE SCR, $p<.014$; SDE pupil dilation, $p<.006$; NDE rating, $p<.028$; NDE SCR, $p<.021$; NDE pupil dilation, $p<.003$. Pairwise comparisons were used only to follow up significant 2-way interactions. In the case of a three-way interaction with IU or STICSA, pairwise comparisons were examined from a 2-way interaction.

In the MLMs two continuous predictor variables were entered (IU, STICSA), a significant interaction with one variable but not the other suggests specificity. Based on our prior work, we expected specificity for IU, but we explored interactions with STICSA, given extant findings with trait anxiety in the conditioning literature (Lonsdorf \& Merz, 2017). Where a significant interaction was observed with IU or STICSA, we performed follow-up pairwise comparisons on the estimated marginal means of the relevant conditions estimated at specific IU values of + or -1 SD of mean IU, adjusted for STICSA (or IU). Similar analyses have been published elsewhere (Morriss, Macdonald, \& van Reekum, 2016; Morriss, McSorley, \& van Reekum, 2017).

We conducted MLMs on SCR magnitude and pupil dilation to check that the middle trials (17-32) for the extended exposure condition and the last trials of the regular exposure condition (17-32) were comparable during SDE. For this analysis, we entered Condition (Regular Exposure, Extended Exposure), Stimulus (CS+, CS-) and Trial (16 trials for CS+ and CS- (last 8 CS+/CS- for Regular Exposure, middle 8 CS+/CS- for Extended Exposure) at level 1 and individual subjects at level 2. 
We included additional MLMs to assess SCR magnitude and pupil dilation across trials during SDE and NDE. We entered Condition (Regular Exposure, Extended Exposure), Stimulus (CS+, CS-) and Trial (16 trials for CS+ and CS- (first 8 , last 8)) at level 1 and individual subjects at level 2.

\section{Results}

For descriptive statistics see Table 1.

\section{Questionnaires}

The self-reported anxiety measures were normally distributed and had good internal reliability (see Figure 2): IU $(M=65.79, S D=20.12$, range $=32-125, \alpha=.94)$; STICSA $(M=40.59, S D=9.58$, range $=22-69, \alpha=.87)$. IU was positively significantly correlated with STICSA, $r(142)=.682, p<.001$. The regular and extended conditions had a similar range of scores for the IU and STICSA measures (Regular IU: $M=65.59, S D=20.33$; Extended IU: $M=66.02, S D=20.02$; Regular STICSA: $M=39.36, S D=9.47$; Extended STICSA: $M=41.95, S D=9.59$ ).

The regular and extended conditions also had a similar range of scores for the shortened version of the Intolerance of Uncertainty Scale (IUS-12: Carleton, Norton \& Asmundson, 2007) and the Prospective and Inhibitory Intolerance of Uncertainty (P-IU, I-IU) subscales (Regular IUS-12: $M=29.55, S D=8.50$; Extended IUS-12: $M=$ 31.29, $S D=10.08$; Regular P-IU: $M=18.89, S D=5.39$; Extended P-IU: $M=20.44$, $S D=6.17$; Regular I-IU: $M=10.65, S D=3.98$; Extended I-IU: $M=10.85, S D=$ 4.57).

\section{Ratings}


The sound stimulus was rated as aversive $(M=2.44 S D=1,17$ range $1-5$, where $1=$ very negative and $9=$ very positive $)$ and arousing $(M=6.65, S D=1.56$, range where $1=$ calm and $9=$ excited). No significant differences between the regular exposure condition and extended exposure condition were found for the valence and arousal ratings of the sound, $p$ 's $>.4$.

Higher expectancy ratings of the sound with the CS+ versus CS- was found during acquisition [Stimulus, $F(1,143)=938.011, p<.001$, see Table 1 and Figure 3A]. No other significant main effects of Condition or interactions with STICSA or IU were found for the expectancy ratings during acquisition, $\max F=3.358$.

During SDE, participants displayed higher expectancy ratings of the sound with the CS+ versus CS-, $p<.001$. Expectancy ratings of the sound with the CS+ dropped over time, $p s<.001$ [Stimulus, $F(1,334.025)=267.902, p<.001$; Time, $F(1,334.025)=44.012, p<.001$; Stimulus $x$ Time, $F(1,334.025)=19.880, p<.001]$. No other significant main effects of Condition or interactions with STICSA or IU were found for the expectancy ratings during SDE, max $F=1.524$.

A similar pattern was observed during NDE, whereby participants displayed higher expectancy ratings of the sound with the CS+ versus CS-, $p<.001$. Expectancy ratings of the sound with the CS+ and CS- dropped over time, $p s<.001$ [Stimulus, $F(1,289.705)=119.682, p<.001$; Time, $F(1,289.705)=31.836, p<$ .001 ; Stimulus $x$ Time, $F(1,289.705)=4.872, p=.028]$. During NDE, expectancy ratings were higher overall for the regular exposure condition $(M=2.57, S D=1.38)$ compared to the extended exposure condition $(M=1.99, S D=1.39), p=.015$ [Condition, $F(1,152.357)=5.998, p=.015]$.

Individual differences in IU were related to expectancy ratings during NDE [Condition $\times$ Time $\times$ IU, $F(1,289.705)=4.056, p=.045$; see Figure 4]. This 
significant interaction for expectancy ratings suggested that individuals with lower and higher IU benefited from extended exposure, relative to their counterparts in the regular exposure condition. Expectancy ratings were smaller for both CS+ and CSacross early to late NDE for Individuals with lower IU in the regular exposure condition, $p=.357$, whilst expectancy ratings to the CS+ and CS- dropped further across early to late NDE for Individuals with lower IU in the extended exposure condition, $p<.001$. Expectancy ratings to the CS+ and CS- were larger in early vs. late NDE for individuals with higher IU in the regular exposure condition, $p<.001$, whilst expectancy ratings were smaller to the CS+ and CS- during early and late NDE for Individuals with higher IU in the extended exposure condition, $p=.231$. No other significant main effects of Condition or interactions with STICSA or IU were found for the expectancy ratings during NDE, $\max F=1.751$.

\section{SCR}

SCR was significantly higher to the CS+ vs. CS- during acquisition [Stimulus, $F(1,129)=37.455, p<.001$; see Table 1 and Figure 3B]. No other significant main effects of Condition or interactions with STICSA or IU were observed for SCR during acquisition, $\max F=894$.

During SDE, SCR was higher to the CS+ versus CS-, $p<.001$. In addition, SCR to both the CS+ and CS- dropped over time, $p=.003$ [Stimulus, $F(1,491.582)$ $=29.260, p<.001 ;$ Time, $F(1,491.582)=8.610, p<.001]$. The interaction between Condition $\mathrm{x}$ Stimulus $\mathrm{x}$ IU was not significant [Condition $\mathrm{x}$ Stimulus $\mathrm{x}$ Time $\mathrm{x} I U, F(1$, 491.582) $=2.990, p=.084]$.

Individual differences in STICSA were related to SCR during SDE [Condition $\mathrm{x}$ Time $\times$ STICSA, $F(1,491.582)=5.220, p=.023$; see Figure 5]. Participants with 
lower STICSA scores in the extended exposure condition showed a significant reduction in SCR response from early to late SDE, $p=.005$, whilst all other condition and STICSA combinations showed no reduction in SCR from early to late SDE, ps > .05. No other significant main effects of Condition or interactions with STICSA or IU were found for SCR during SDE, $\max F=3.745$.

During NDE, SCR was higher to the CS+ versus CS- during early extinction, $p$ $<.001$, but no difference was observed for the CS+ and CS- during late extinction $p$ $=.073$ [Stimulus, $F(1,447.028)=29.794, p<.001 ;$ Time, $F(1,447.028)=51.149, p$ $<.001 ;$ Stimulus $\times$ Time, $F(1,447.028)=9.072, p=.003]$.

Individual differences in STICSA were related to SCR during NDE [Condition $x$ Time $x$ STICSA, $F(1,447.028)=4.428, p=.036$ : see Figure 5]. Participants with higher STICSA scores in the extended exposure condition had lower SCR response during the early and late parts of the NDE phase, $p>.05$, whilst all other condition and STICSA combinations showed a reduction in SCR from early to late NDE, $p s<$ .005 .

No other significant main effects of Condition or interactions with STICSA or IU were found for SCR during NDE, $\max F=1.803$.

\section{Pupil dilation}

During acquisition, no significant main effect of stimulus was observed for pupil dilation [Stimulus, $F(1,607.862)=1.437, p=.231]$.

Pupil dilation was significantly larger to the CS+ vs. CS- during SDE [Stimulus, $F(1,2047.009)=27.420, p<.001$; see Table 1 and Figure 3C]. Additional interactions with STICSA were observed for pupil dilation during SDE [Condition $x$ Stimulus $\times$ STICSA, $F(1,2047.009)=4.786, p=.029$; Condition $\times$ Time $\times$ STICSA, 
$F(1,2047.009)=7.356, p=.007]$. Participants with higher STICSA scores in the regular exposure condition had larger pupil dilation to the CS+ vs. CS- during SDE, $p$ $<.001$ (see Figure 6). All other condition and STICSA combinations showed smaller pupil dilation differentiation between CS+ and CS-, p's above the threshold for multiple comparisons $(p=.003)$. In addition, for the extended exposure condition lower STICSA was associated with reduced pupil dilation across early $(M=.025, S D$ $=0.33)$ to late $(\mathrm{M}=-.106, \mathrm{SD}=0.34) \mathrm{SDE}, p=.007$, whilst higher STICSA was associated with an increased pupil dilation across early $(\mathrm{M}=-.052, \mathrm{SD}=0.28)$ to late $(M=.079, S D=0.28), p=.004$. However, these effects were just above the threshold for multiple comparisons $(p=.003)$. No significant differences for STICSA in the regular exposure condition was observed for pupil dilation across early to late SDE, p's > .05.

Pupil dilation was significantly larger to the CS+ vs. CS- during NDE [Stimulus, $F(1,2072.327)=7.543, p=.006$; see Table 1 and Figure $3 C$ ]. During NDE, pupil dilation significantly dropped across early $(\mathrm{M}=.041, \mathrm{SD}=0.18)$ to late $(\mathrm{M}=-.037, \mathrm{SD}=0.16)$ extinction for the regular condition, $p=.005$, but remained lower for early $(\mathrm{M}=.007, \mathrm{SD}=0.15)$ and late $(\mathrm{M}=.017, \mathrm{SD}=0.16)$ extinction for the extended exposure condition, $p=.731$ [Condition $x$ Time, $F(1,2072.327)=6.924, p$ $=.009]$.

During each experimental phase, pupil dilation was greatest at 1 second post stimulus onset and smallest at 2 seconds post stimulus onset [Acquisition, Second, $F(1,432.306)=164.374, p<.001 ;$ SDE, Second $F(1,1043.920)=310.082, p<.001$; 
NDE, Second $F(1,1026.885)=308.076, p<.001]$. No other significant main effects of Condition, Time, or interactions with IU or STICSA were found, $\max F=2.309^{1}$.

\section{Preregistered manipulation check}

We conducted MLMs on SCR magnitude and pupil dilation to check that the middle trials (17-32) for the extended exposure condition and the last trials of the regular exposure condition (17-32) were comparable during SDE. A significant interaction between Condition and Trial for pupil dilation was observed during SDE, where larger pupil dilation was found for the first trial for the extended exposure condition vs. the regular exposure condition [Condition $\times$ Trial, $F(1,529.759)=1.938, p=$ .018]. No other significant main effects or interactions between Stimulus, Trial and Condition were observed for SCR and pupil dilation during SDE, Max $F=1.662$. These results suggest that the late trials from the regular exposure condition and middle trials from the extended exposure condition were comparable.

\section{Preregistered analyses by trial}

We included additional MLMs to assess SCR magnitude and pupil dilation across trials during SDE and NDE. A significant interaction between Condition and Trial for pupil dilation was observed during NDE, where larger pupil dilation was found for the first two trials for the regular exposure condition vs. the extended exposure condition [Condition $\mathrm{x}$ Trial, $F(1,444.448)=1.898, p=.022]$. No other significant main effects

\footnotetext{
${ }^{1}$ Additional interactions with IU were observed for pupil dilation during NDE [Condition $\times$ Stimulus $\times$ IU, $F(1,2072.327)=4.023, p=.045$; Condition $\times$ Stimulus $\mathrm{x}$ Time $x$ IU, $F(1,2072.327)=4.266, p=.039]$. However, these interactions were not significant when IU was entered into the model alone, suggesting these results as potentially spurious [Condition $\times$ Stimulus $x$ IU, $F(1,2049.829)=1.135, p=.287$; Condition $\times$ Stimulus $\times$ Time $\times$ IU, $F(1,2049.829)=.771, p=.380]$.
} 
or interactions between Trial, Stimulus and Condition were observed for SDE or NDE, Max $F=1.680$.

\section{Additional preregistered exploratory analyses with the IUS-12 and its subscales}

To examine whether safety-learning and -retention were related to the IUS-12, P-IU and I-IU scales, we conducted further exploratory analyses. We created the following difference scores for each measure (ratings, SCR and pupil dilation): [Acquisition CS+ - CS-], [SDE early CS+ - CS-], [SDE late CS+ - CS-], [SDE early CS+ - CS- SDE late CS+ - CS-], [NDE early CS+ - CS-], [NDE late CS+ - CS-], [NDE early CS+ - CS- - SDE late CS+ - CS-]. We examined whether the difference scores were correlated with the IUS-12, P-IU, and I-IU by Condition (Regular, Extended).

To assess specificity between Condition, we tested the significance of the difference between the two correlation coefficients i.e. a correlation for regular exposure vs. extended exposure. Then, to assess specificity of the relevant IUS measure over STICSA, we conducted partial correlations and tested the significance of the difference between the two partial correlation coefficients. We only report the effects that survive the test of significant difference between the two Condition (Regular, Extended) correlation coefficients.

There was a significant correlation between the SCR difference score (NDE early CS+ - CS- - SDE late CS+ - CS-) and I-IU in the regular exposure condition, $r(61)=.322, p=.01$ but not for extended exposure condition, $r(55)=-.235, p>.05$. The difference between the correlations for the regular and extended exposure conditions was significant, $z=3.06, p=.002$. Moreover, when controlling for 
STICSA, the difference between the partial correlations for the regular and extended exposure conditions remained significant, $z=3.05, p=.002$.

The effect above was driven by differences in SCR during the early part of $\mathrm{NDE}$, as there was also significant correlation between the SCR difference score (NDE early CS + - CS-) and I-IU in the regular exposure condition, $r(61)=.297, p=$ .018 but not for extended exposure condition, $r(55)=-.146, p>.05$. The difference between the correlations for the regular and extended exposure conditions was significant, $z=2.42, p=.015$. Again, when controlling for STICSA, the difference between the partial correlations for the regular and extended exposure conditions remained significant, $z=2.4, p=.016^{2}$. In sum, higher IIU scores were associated with greater SCR response during the early part of NDE in the regular exposure condition, compared to the extended exposure condition (see Fig 7).

No other significant correlations were observed between any of the measures and the IUS-12, P-IU, and I-IU during acquisition, SDE and NDE, $p$ 's $>.05$.

\section{Discussion}

Here we examined the effect of self-reported IU and exposure experience on safetylearning and -retention. We failed to replicate previous work showing that IU is associated with poorer safety-learning indexed via SCR. We found preliminary evidence for promoted safety-retention in individuals with higher Inhibitory IU who underwent extended exposure, relative to individuals with higher Inhibitory IU who

\footnotetext{
${ }^{2}$ We decided to do correlational analyses rather than MLMs because of the number of tests required for each dependent variable and phase of the experiment i.e MLMs with IUS-12 and each subscale alone, as well as with STICSA included. A similar result was found for SCR magnitude during NDE when I-IU was entered alone into the MLM [Condition $\times$ Stimulus $\times$ Time $\times I-I U, F(1,442.225)=10.435, p=.001]$ and with STICSA included in the MLM [Condition $x$ Stimulus $\times$ Time $\times$ I-IU, F $(1,452.236)$ $=10.319, p=.001]$.
} 
underwent regular exposure, indexed via SCR. These findings further our current understanding of the role of IU in safety-learning and -retention, informing models of IU and exposure-based treatments.

We observed typical patterns of conditioning in the acquisition, SDE and NDE phases: SCR magnitude and expectancy ratings were higher for the learned threat vs. safety cues. There was no significant reduction in responding to the learned threat vs. safety cues across the SDE phase for SCR magnitude, pupil dilation and expectancy ratings. However, we observed a reduction in responding to the learned threat vs. safety cues across the NDE phase for SCR magnitude and expectancy ratings. The absence of safety-learning across SDE may have occurred because a partial reinforcement schedule was used during the acquisition phase, which is known to prolong conditioning (Grady et al., 2016; Leonard, 1975).

We observed no significant differences between the extended exposure condition vs. regular exposure condition during SDE on any of the measures. However, the extended exposure condition vs. the regular exposure condition showed lower pupil dilation and expectancy ratings overall during NDE. Such findings suggest that exposure length may not impact safety-learning directly after an aversive event but may inhibit anxious behaviours the next day i.e. generally reduce arousal and expectancy of threat. Our findings are at odds with previous research, which has shown that more trials versus fewer trials during SDE results in greater safety-learning, indexed via reduced startle blink magnitude and expectancy ratings (Golkar et al., 2013; Prenoveau, Craske, Liao, \& Ornitz, 2013). Unfortunately, only a few human studies have examined trial number during SDE (Golkar et al., 2013; Prenoveau et al., 2013), and as far as we are aware no human studies have examined the impact of trial number of SDE upon NDE. The findings from the current 
study provide some preliminary evidence that extended exposure may be beneficial, but also highlight that further work is needed in order to understand how exposure length impacts safety-learning and -retention mechanisms.

Past research has shown that higher IU is associated with reduced safetylearning, indexed by greater SCR, greater corrugator supercilii activity and pupil dilation to cues that no longer signal threat during the late part of SDE (Morriss, 2019; Morriss et al., 2015; Morriss, Christakou, et al., 2016; Morriss et al., 2019; Morriss \& van Reekum, 2019). Whilst effects of IU during SDE on SCR have been replicated many times (6 out of 7 experiments), in the current experiment it failed.

Based on prior experiments (Dunsmoor et al., 2015; Lucas et al., 2018), we hypothesised that during NDE higher IU would be associated with poorer safetyretention in the regular exposure condition vs. the extended exposure condition. We did not observe significant differences in safety-retention based on IU and exposure condition for SCR and pupil dilation. For expectancy ratings, we found individuals with higher IU in the regular exposure condition displayed greater expectancy ratings of the sound with both the CS+ and CS- during early vs. late NDE. In comparison, individuals with higher IU in the extended exposure condition and lower IU in both the regular and extended exposure conditions had lower expectancy ratings of the sound with both the CS+ and CS- throughout NDE.

The lack of effects with the full 27 item IU scale during SDE and NDE may have occurred for a number of reasons. Firstly, in previous studies, IU has been used against other measures of self-reported anxiety (trait anxiety and worry) and not STICSA, which has been shown as a purer measure of self-reported anxiety (Grös et al., 2007). Secondly, whilst the distribution of IU and STICSA were similar in the regular and extended exposure conditions, other individual differences factors 
that were not controlled for may have influenced the results (Lonsdorf \& Merz, 2017) i.e. biological sex, use of contraceptive, use of anxiolytic medications and prior mental health history.

Recent IU research typically relies on the 12 item IU scale and its subscales (Prospective: anticipation of uncertain threat; Inhibitory: paralysis under uncertainty), over the full 27 item scale, given its consistent and robust psychometrics (Carleton, Norton, \& Asmundson, 2007; Hong \& Lee, 2015; Khawaja \& Yu, 2010). Therefore, we conducted preregistered exploratory analyses with the IUS-12 and its subscales. No significant associations were found between extinction learning metrics and the the IUS-12 total score or Prospective IU subscale. However, higher Inhibitory IU in the regular exposure condition, relative to higher Inhibitory IU in the extended exposure condition, was associated with poorer safety-retention during early NDE, indexed via SCR. Moreover, this association during early NDE was specific to Inhibitory IU over STICSA. Notably, this result with Inhibitory IU is in line with previous work showing that higher IU is associated with poorer safety-retention during the start of NDE (Dunsmoor et al., 2015) and reinstatement (Lucas et al., 2018). However, the specificity of IU or its subscales was not examined in past work and therefore it is not known whether these findings were driven by the inhibitory component of IU. Nonetheless, the current study highlights that individuals with higher Inhibitory IU may benefit from extended exposure. Interestingly, Inhibitory IU is uniquely associated with symptoms in a number of disorders that rely on exposure therapy, such as social phobia, panic disorder, agoraphobia and post-traumatic stress disorder (Fetzner, Horswill, Boelen, \& Carleton, 2013; Mahoney \& McEvoy, 2012; McEvoy \& Mahoney, 2011, 2012). It will be important therefore to replicate the 
effect of Inhibitory IU on NDE, as such work may have relevance for understanding the role of Inhibitory IU in exposure-based treatments (Knowles \& Olatunji, 2018).

Unexpectedly, we found additional results with the STICSA measure. Higher STICSA scores in the regular exposure condition was associated with larger pupil dilation to the learned threat vs. safety cues during SDE. All other condition and STICSA combinations showed no pupil dilation differentiation between the learned threat and safety cues during SDE. Furthermore, STICSA was associated with changes in SCR generally across SDE and NDE. We would have expected specificity of IU, over STICSA. In previous safety-learning research, IU has shown specificity over self-reported trait anxiety and worry (Morriss, Macdonald, et al., 2016; Morriss \& van Reekum, 2019). However, these results suggest that STICSA may be more closely aligned to safety-learning and -retention than trait anxiety and worry. Further replication of STICSA effects on safety-learning and -retention are needed to assess its importance on these mechanisms and specificity over IU.

In conclusion, the results from this study provide some insight into how IU and exposure experience impact safety-learning and -retention. Tentatively, these results suggest that individuals with higher Inhibitory IU may benefit from extended exposure. Further experimental and clinical research is needed to assess how exposure experience can promote safety-learning and -retention in individuals with higher levels of IU. 


\section{Acknowledgements}

This research was supported by: (1) a NARSAD Young Investigator Grant from the Brain \& Behavior Research Foundation (27567) and an ESRC New Investigator Grant (ES/R01145/1) awarded to Jayne Morriss, and (2) an ESRC Future Leaders Grant (ES/L010119/1) awarded to Helen Dodd. The authors would like to thank Jessica Jarvis, Charlotte Elizabeth, Martina Mutti, Laura Bucher and Nicolo Biagi for their help in data collection and data preprocessing. For access to the data please refer to the data supplement provided with the article.

\section{Declaration of interest statement}

The authors declare no conflict of interest. 


\section{References}

Bandelow, B., Michaelis, S., \& Wedekind, D. (2017). Treatment of anxiety disorders. Dialogues in Clinical Neuroscience, 19(2), 93.

Ben-Shakhar, G. (1985). Standardization within individuals: A simple method to neutralize individual differences in skin conductance. Psychophysiology, 22(3), 292-299.

Benjamini, Y., \& Hochberg, Y. (1995). Controlling the false discovery rate: a practical and powerful approach to multiple testing. Journal of the Royal Statistical Society. Series B (Methodological), 289-300.

Bouton, M. E. (2002). Context, ambiguity, and unlearning: Sources of relapse after behavioral extinction. Biological Psychiatry, 52(10), 976-986.

Carleton, R. N. (2016a). Fear of the unknown: One fear to rule them all? Journal of Anxiety Disorders, 41, 5-21.

Carleton, R. N. (2016b). Into the unknown: A review and synthesis of contemporary models involving uncertainty. Journal of Anxiety Disorders, 39, 30-43.

Carleton, R. N., Norton, M. P. J., \& Asmundson, G. J. (2007). Fearing the unknown: A short version of the Intolerance of Uncertainty Scale. Journal of Anxiety Disorders, 21(1), 105-117.

Craske, M. G., Treanor, M., Conway, C. C., Zbozinek, T., \& Vervliet, B. (2014). Maximizing exposure therapy: an inhibitory learning approach. Behaviour Research and Therapy, 58, 10-23.

Dawson, M. E., Schell, A. M., \& Filion, D. L. (2000). The Electrodermal System. In J. T. Cacioppo, L. G. Tassinary, \& G. G. Berntson (Eds.), Handbook of Physiology (2nd ed., pp. 200-223). Cambridge, UK: Cambridge University Press. 
Dugas, M. J., Buhr, K., \& Ladouceur, R. (2004). The Role of Intolerance of Uncertainty in Etiology and Maintenance of Generalized Anxiety Disorder. In: R. G. Heimberg, C. L. Turk, \& D. S. Mennin (Eds.),Generalized anxiety disorder: advances in research and practice (pp. 143-163). New York:Guilford Press.

Dunsmoor, J. E., Campese, V. D., Ceceli, A. O., LeDoux, J. E., \& Phelps, E. A. (2015). Novelty-facilitated extinction: providing a novel outcome in place of an expected threat diminishes recovery of defensive responses. Biological Psychiatry, 78(3), 203-209.

Fetzner, M. G., Horswill, S. C., Boelen, P. A., \& Carleton, R. N. (2013). Intolerance of uncertainty and PTSD symptoms: Exploring the construct relationship in a community sample with a heterogeneous trauma history. Cognitive Therapy and Research, 37(4), 725-734.

Freeston, M. H., Rhéaume, J., Letarte, H., Dugas, M. J., \& Ladouceur, R. (1994). Why do people worry? Personality and Individual Differences, 17(6), 791-802.

Gentes, E. L., \& Ruscio, A. M. (2011). A meta-analysis of the relation of intolerance of uncertainty to symptoms of generalized anxiety disorder, major depressive disorder, and obsessive-compulsive disorder. Clinical Psychology Review, 31(6), 923-933.

Golkar, A., Bellander, M., \& Öhman, A. (2013). Temporal properties of fear extinction—Does time matter? Behavioral Neuroscience, 127(1), 59.

Grady, A. K., Bowen, K. H., Hyde, A. T., Totsch, S. K., \& Knight, D. C. (2016). Effect of continuous and partial reinforcement on the acquisition and extinction of human conditioned fear. Behavioral Neuroscience, 130(1), 36. 
Grös, D. F., Antony, M. M., Simms, L. J., \& McCabe, R. E. (2007). Psychometric properties of the State-Trait Inventory for Cognitive and Somatic Anxiety (STICSA): comparison to the State-Trait Anxiety Inventory (STAI). Psychological Assessment, 19(4), 369.

Grupe, D. W., \& Nitschke, J. B. (2013). Uncertainty and anticipation in anxiety: an integrated neurobiological and psychological perspective. Nature Reviews Neuroscience, 14(7), 488-501.

Hong, R. Y., \& Lee, S. S. (2015). Further clarifying prospective and inhibitory intolerance of uncertainty: Factorial and construct validity of test scores from the Intolerance of Uncertainty Scale. Psychological Assessment, 27(2), 605.

Khawaja, N. G., \& Yu, L. N. H. (2010). A comparison of the 27-item and 12-item intolerance of uncertainty scales. Clinical Psychologist, 14(3), 97-106.

Knowles, K. A., \& Olatunji, B. O. (2018). Enhancing Inhibitory Learning: The Utility of Variability in Exposure. Cognitive and Behavioral Practice, 26(1), 186-200.

Leonard, D. W. (1975). Partial reinforcement effects in classical aversive conditioning in rabbits and human beings. Journal of Comparative and Physiological Psychology, 88(2), 596.

Leuchs, L., Schneider, M., \& Spoormaker, V. I. (2019). Measuring the conditioned response: A comparison of pupillometry, skin conductance, and startle electromyography. Psychophysiology, 56(1), e13283.

Lonsdorf, T. B., Menz, M. M., Andreatta, M., Fullana, M. A., Golkar, A., Haaker, J., . . . Kruse, O. (2017). Don't fear 'fear conditioning': Methodological considerations for the design and analysis of studies on human fear acquisition, extinction, and return of fear. Neuroscience \& Biobehavioral Reviews, 77, 247-285. 
Lonsdorf, T. B., \& Merz, C. J. (2017). More than just noise: Inter-individual differences in fear acquisition, extinction and return of fear in humansBiological, experiential, temperamental factors, and methodological pitfalls. Neuroscience \& Biobehavioral Reviews, 80, 703-728.

Lonsdorf, T. B., Merz, C. J., \& Fullana, M. A. (2019). Fear extinction retention-is it what we think it is? Biological Psychiatry, 85(12), 1074-1082.

Lucas, K., Luck, C. C., \& Lipp, O. V. (2018). Novelty-facilitated extinction and the reinstatement of conditional human fear. Behaviour Research and Therapy, 109, 68-74.

Mahoney, A. E., \& McEvoy, P. M. (2012). A transdiagnostic examination of intolerance of uncertainty across anxiety and depressive disorders. Cognitive Behaviour Therapy, 41(3), 212-222.

McEvoy, P. M., \& Mahoney, A. E. (2011). Achieving certainty about the structure of intolerance of uncertainty in a treatment-seeking sample with anxiety and depression. Journal of Anxiety Disorders, 25(1), 112-122.

McEvoy, P. M., \& Mahoney, A. E. (2012). To be sure, to be sure: Intolerance of uncertainty mediates symptoms of various anxiety disorders and depression. Behavior Therapy, 43(3), 533-545.

Milad, M. R., \& Quirk, G. J. (2012). Fear extinction as a model for translational neuroscience: ten years of progress. Annual Review of Psychology, 63, 129151.

Morriss, J. (2019). What do I do now? Intolerance of uncertainty is associated with discrete patterns of anticipatory physiological responding to different contexts. Psychophysiology, 56(9), e13396. 
Morriss, J., Chapman, C., Tomlinson, S., \& Van Reekum, C. M. (2018). Escape the bear and fall to the lion: The impact of avoidance availability on threat acquisition and extinction. Biological Psychology, 138, 73-80.

Morriss, J., Christakou, A., \& Van Reekum, C. M. (2015). Intolerance of uncertainty predicts fear extinction in amygdala-ventromedial prefrontal cortical circuitry. Biology of Mood \& Anxiety Disorders, 5(1), 1.

Morriss, J., Christakou, A., \& Van Reekum, C. M. (2016). Nothing is safe: Intolerance of uncertainty is associated with compromised fear extinction learning. Biological Psychology, 121, 187-193.

Morriss, J., Macdonald, B., \& van Reekum, C. M. (2016). What Is Going On Around Here? Intolerance of Uncertainty Predicts Threat Generalization. PloS one, 11(5), e0154494.

Morriss, J., McSorley, E., \& van Reekum, C. M. (2017). I don't know where to look: the impact of intolerance of uncertainty on saccades towards non-predictive emotional face distractors. Cognition and Emotion, 1-10.

Morriss, J., Saldarini, F., Chapman, C., Pollard, M., \& van Reekum, C. M. (2018). Out with the old and in with the new: The role of intolerance of uncertainty in reversal of threat and safety. Journal of Experimental Psychopathology, 10(1), 2043808719834451.

Morriss, J., Saldarini, F., \& Van Reekum, C. M. (2019). The role of threat level and intolerance of uncertainty in extinction. International Journal of Psychophysiology, 142, 1-9.

Morriss, J., \& van Reekum, C. M. (2019). I feel safe when i know: Contingency instruction promotes threat extinction in high intolerance of uncertainty individuals. Behaviour research and Therapy, 116, 111-118. 
Neumann, D. L., \& Waters, A. M. (2006). The use of an unpleasant sound as an unconditional stimulus in a human aversive Pavlovian conditioning procedure. Biological Psychology, 73(2), 175-185.

Peugh, J. L. (2010). A practical guide to multilevel modeling. Journal of School Psychology, 48(1), 85-112.

Prenoveau, J. M., Craske, M. G., Liao, B., \& Ornitz, E. M. (2013). Human fear conditioning and extinction: Timing is everything... or is it? Biological Psychology, 92(1), 59-68.

Quené, H., \& Van den Bergh, H. (2004). On multi-level modeling of data from repeated measures designs: A tutorial. Speech Communication, 43(1), 103121.

Rabinak, C. A., Angstadt, M., Lyons, M., Mori, S., Milad, M. R., Liberzon, I., \& Phan, K. L. (2014). Cannabinoid modulation of prefrontal-limbic activation during fear extinction learning and recall in humans. Neurobiology of Learning and Memory, 113, 125-134.

Ree, M. J., French, D., MacLeod, C., \& Locke, V. (2008). Distinguishing cognitive and somatic dimensions of state and trait anxiety: Development and validation of the State-Trait Inventory for Cognitive and Somatic Anxiety (STICSA). Behavioural and Cognitive Psychotherapy, 36(3), 313-332.

Sehlmeyer, C., Dannlowski, U., Schöning, S., Kugel, H., Pyka, M., Pfleiderer, B., . . . Arolt, V. (2011). Neural correlates of trait anxiety in fear extinction. Psychological Medicine, 41(04), 789-798.

Shin, L. M., \& Liberzon, I. (2009). The neurocircuitry of fear, stress, and anxiety disorders. Neuropsychopharmacology, 35(1), 169-191. 
Snijders, T. A. (2005). Power and sample size in multilevel linear models.

Encyclopedia of Statistics in Behavioral Science.

Wicking, M., Steiger, F., Nees, F., Diener, S. J., Grimm, O., Ruttorf, M., . . Flor, H. (2016). Deficient fear extinction memory in posttraumatic stress disorder. Neurobiology of Learning and Memory, 136, 116-126.

Xia, W., Dymond, S., Lloyd, K., \& Vervliet, B. (2017). Partial reinforcement of avoidance and resistance to extinction in humans. Behaviour Research and Therapy, 96, 79-89. 


\section{Figure Captions}

Figure 1. Image displaying experimental conditions and procedure.

Figure 2. Histograms of IU and STICSA self-report measures by condition.

Figure 3. Bar graphs depicting mean expectancy ratings (A), SCR magnitude (B) and pupil dilation $(\mathrm{C})$ for each condition and stimulus type during each experimental phase. Error bars represent standard error. Expectancy ratings, $1=$ Don't expect, $9=$ Do expect. Square root transformed and z-transformed SCR magnitude ( $\mu S)$, skin conductance magnitude measured in microSiemens. Z-scored pupil dilation and measured in $\Delta \mathrm{mm}$.

Figure 4. Bar graphs depicting IU estimated at + or -1 SD of the mean (controlling for STICSA) by condition and time for expectancy ratings during next-day extinction. Expectancy ratings were higher in early vs. late NDE for individuals with higher IU in the regular exposure condition, whilst expectancy ratings were smaller during early and late NDE for individuals with lower and higher IU in all other conditions. Error bars represent standard error. Expectancy ratings, $1=$ Don't expect, $9=$ Do expect.

Figure 5. Bar graphs showing STICSA estimated at + or - 1 SD of the mean (controlling for IU) by condition and time for SCR during same-day extinction and next-day extinction. Lower STICSA scores in the extended exposure condition was associated with a reduction in SCR response from early to late SDE, whilst all other condition and STICSA combinations showed no reduction in SCR from early to late SDE. Higher STICSA scores in the extended exposure condition had lower SCR 
response during the early and late parts of the NDE phase, whilst all other condition and STICSA combinations showed a reduction in SCR from early to late NDE. Error bars represent standard error. Square root transformed and z-transformed SCR magnitude $(\mu \mathrm{S})$, skin conductance magnitude measured in microSiemens.

Figure 6. Bar graphs showing effects of STICSA estimated at + or - 1 SD of the mean (controlling for IU) by condition and stimulus for pupil dilation during same-day extinction. Higher STICSA scores in the regular exposure condition was associated with larger pupil dilation to the CS+ vs. CS- during SDE. All other condition and STICSA combinations showed smaller pupil dilation differentiation between CS+ and CS-. Error bars represent standard error. Z-scored pupil dilation and measured in $\Delta \mathrm{mm}$.

Figure 7. Scatter plots displaying correlations by condition between SCR magnitude CS+ - CS- difference scores and Inhibitory IU for early NDE. Positive difference scores indicate larger SCR magnitude to the CS+ vs. CS-. Higher Inhibitory IU was associated with larger SCR magnitude to the CS+ vs. CS- in the early part of NDE for the regular exposure condition, compared to the extended exposure condition. Square root transformed and z-transformed SCR magnitude $(\mu S)$, skin conductance magnitude measured in microSiemens. 
Table 1. Summary of means (SD) for each dependent measure as a function of condition (CS+ and CS-), separately for acquisition, early extinction and late extinction.

\begin{tabular}{|c|c|c|c|c|c|c|c|c|c|c|}
\hline \multirow[t]{3}{*}{ Measure } & \multicolumn{6}{|c|}{ Day 1} & \multicolumn{4}{|c|}{ Day 2} \\
\hline & \multicolumn{2}{|c|}{ Acquisition } & \multicolumn{2}{|c|}{ Early Extinction } & \multicolumn{2}{|c|}{ Late Extinction } & \multicolumn{2}{|c|}{ Early Extinction } & \multicolumn{2}{|c|}{ Late Extinction } \\
\hline & $\mathrm{CS}_{+}$ & CS- & $\mathrm{CS}_{+}$ & CS- & CS+ & CS- & $\mathrm{CS}_{+}$ & CS- & $\mathrm{CS}+$ & CS- \\
\hline Expectancy rating (1-9) & 6.59 & 2.11 & 4.48 & 2.01 & 3.18 & 1.76 & 3.13 & 1.93 & 2.41 & 1.64 \\
\hline \multirow[t]{2}{*}{ Square root transformed and z-scored SCR magnitude $(\sqrt{ } \mu \mathrm{s})$} & 0.27 & 0.00 & 0.06 & -0.10 & -0.02 & -0.15 & 0.21 & 0.00 & -0.07 & -0.13 \\
\hline & $(0.51)$ & $(0.30)$ & $(0.39)$ & $(0.30)$ & $(0.35)$ & (0.39) & $(0.42)$ & $(0.32)$ & (0.29) & $(0.36)$ \\
\hline Z-scored Pupil dilation $(\Delta \mathrm{mm})$ & $(0.55)$ & $(0.46)$ & $(0.31)$ & $(0.27)$ & $(0.32)$ & $(0.34)$ & $(0.36)$ & $(0.34)$ & $(0.34)$ & $(0.33)$ \\
\hline
\end{tabular}

Note: SCR magnitude $(\sqrt{\mu S})$, square root transformed and z-scored skin conductance magnitude measured in microSiemens. Pupil dilation $(\Delta \mathrm{mm})$, z-scored pupil dilation measured in delta millimetres. 


\section{Threat \\ Acquisition}

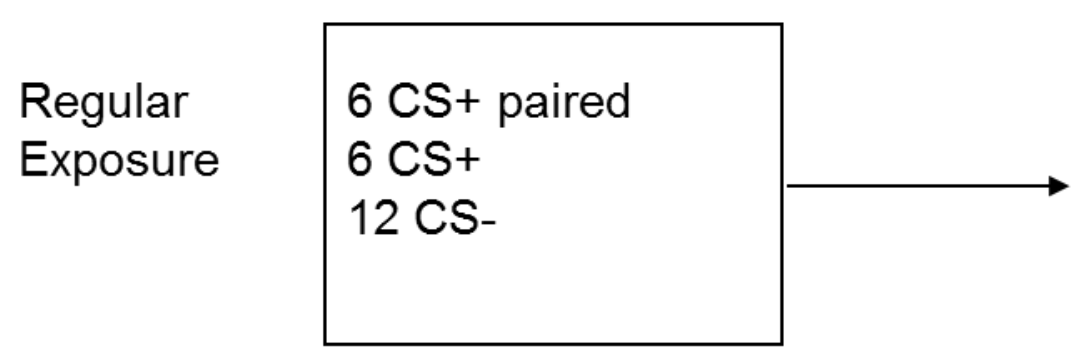

\begin{tabular}{l|l|} 
Extended & $6 \mathrm{CS}+$ paired \\
Exposure & $\mathrm{CS}+$ \\
$12 \mathrm{CS}-$
\end{tabular}

Same-Day

Extinction

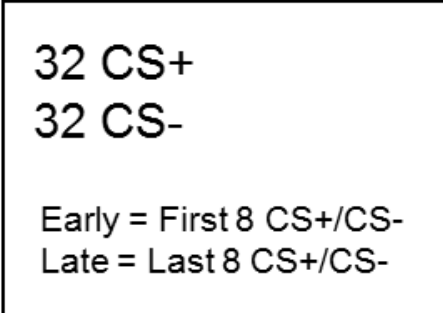

\section{$48 \mathrm{CS}+$} 48 CS-

Early = First $8 \mathrm{CS}+/ \mathrm{CS}$ -

Late $=$ Last $8 \mathrm{CS}+/ \mathrm{CS}$ -
Next-Day

Extinction

$32 \mathrm{CS}+$

$32 \mathrm{CS}-$

Early = First $8 \mathrm{CS}+/ \mathrm{CS}-$

Late $=$ Last $8 \mathrm{CS}+/ \mathrm{CS}-$

$48 \mathrm{CS}+$

$48 \mathrm{CS}$ -

Early = First $8 \mathrm{CS}+/ \mathrm{CS}$ -

Late $=$ Last $8 \mathrm{CS}+/ \mathrm{CS}$ - 
A

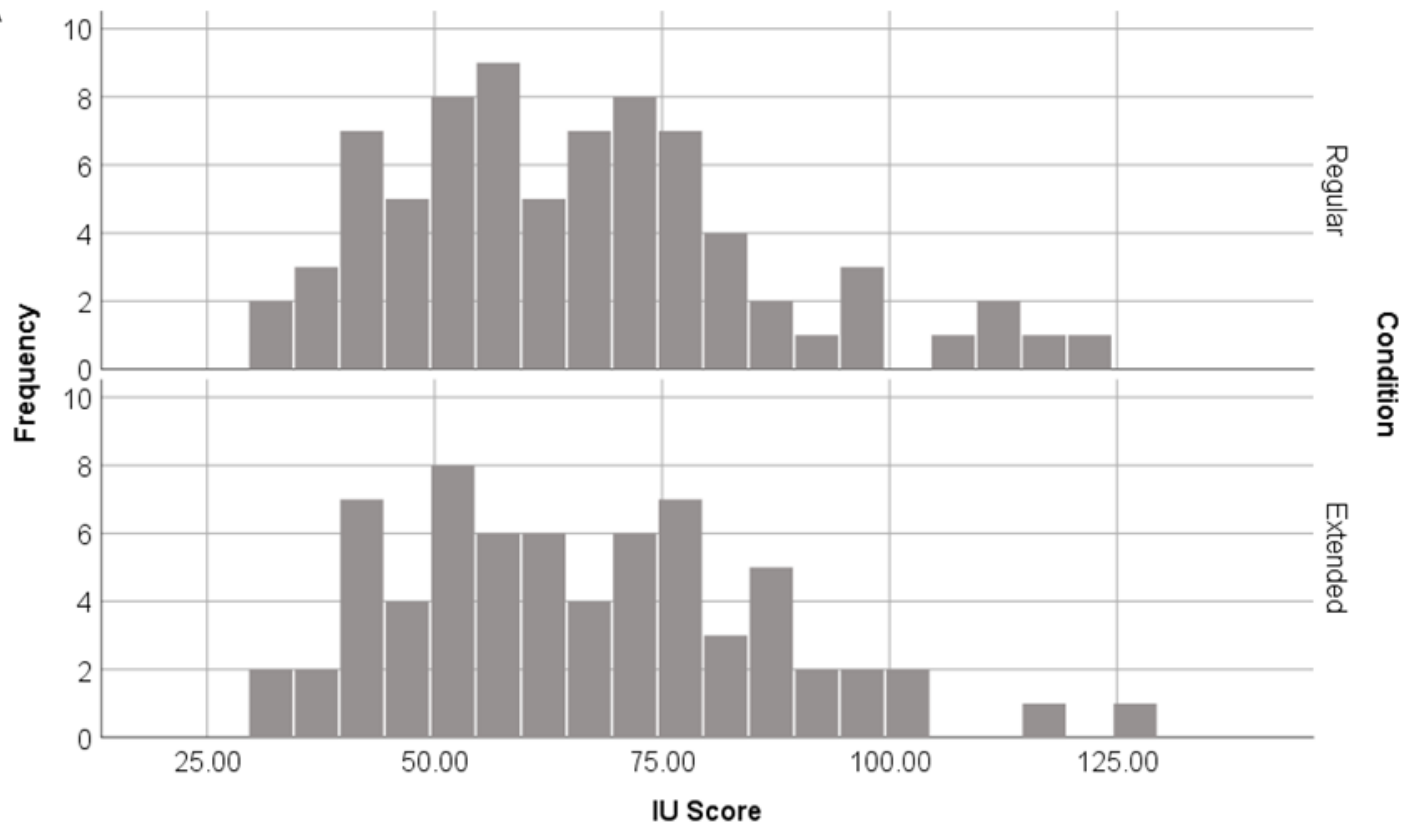

B

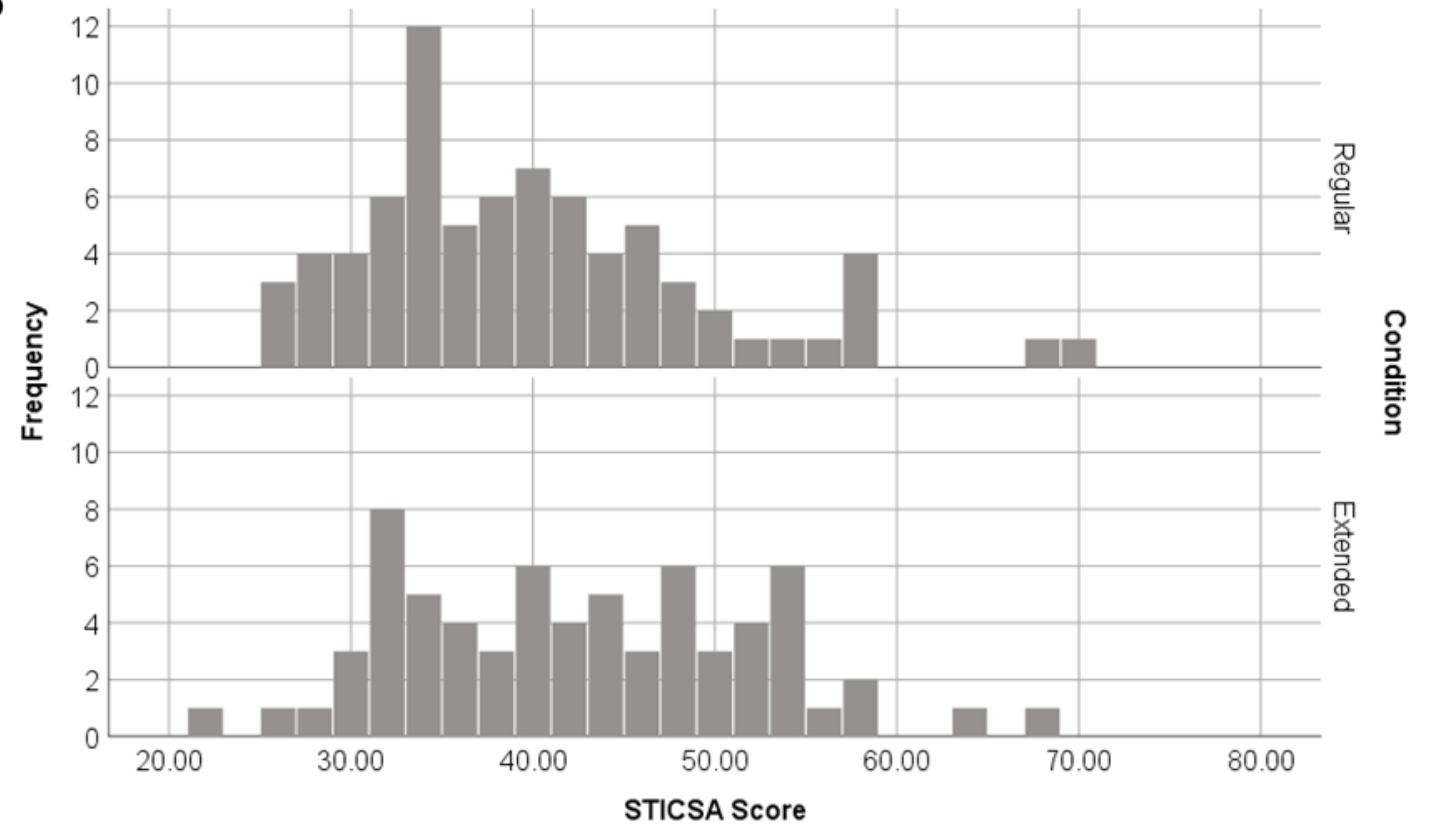




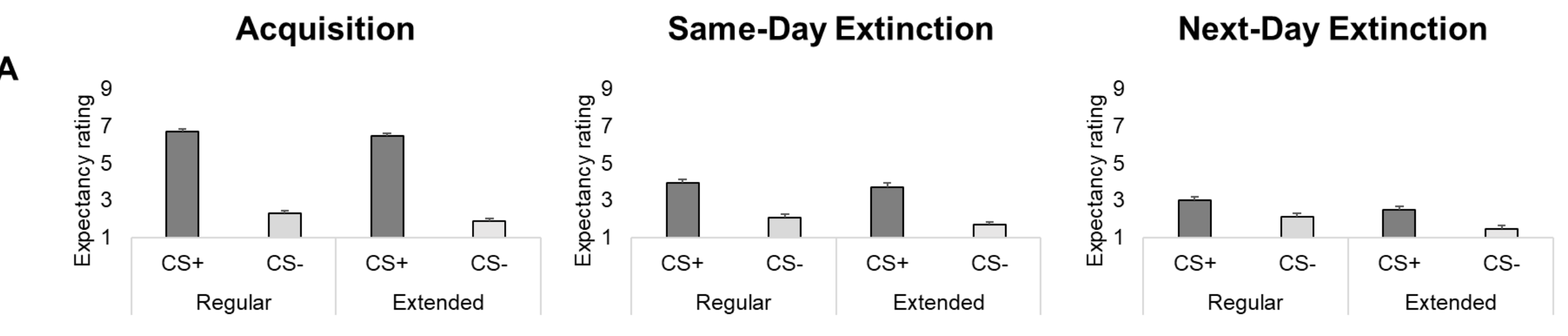

B

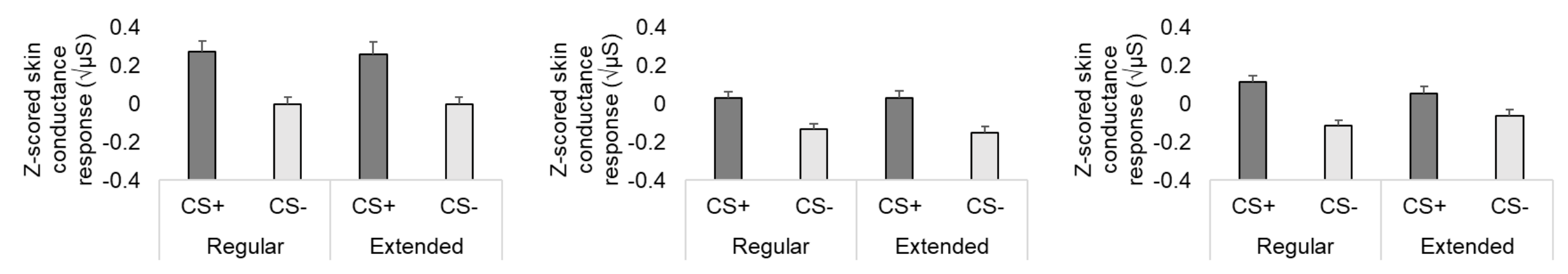

C
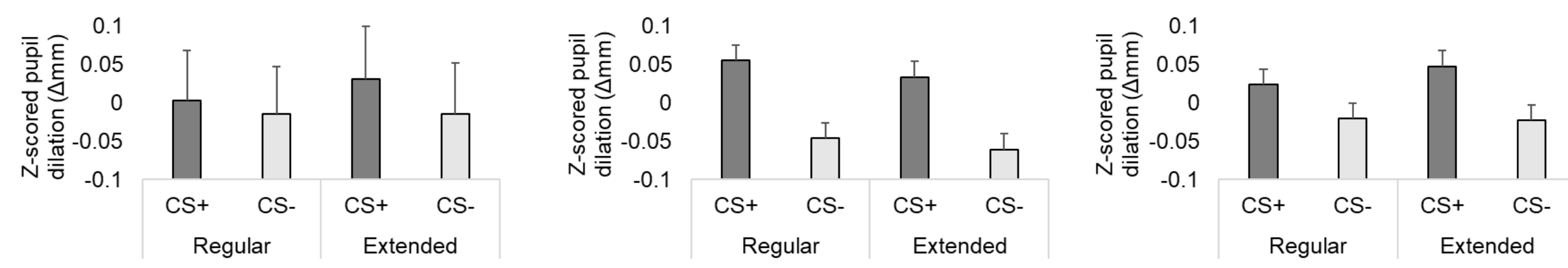


\section{Next-Day Extinction}

9

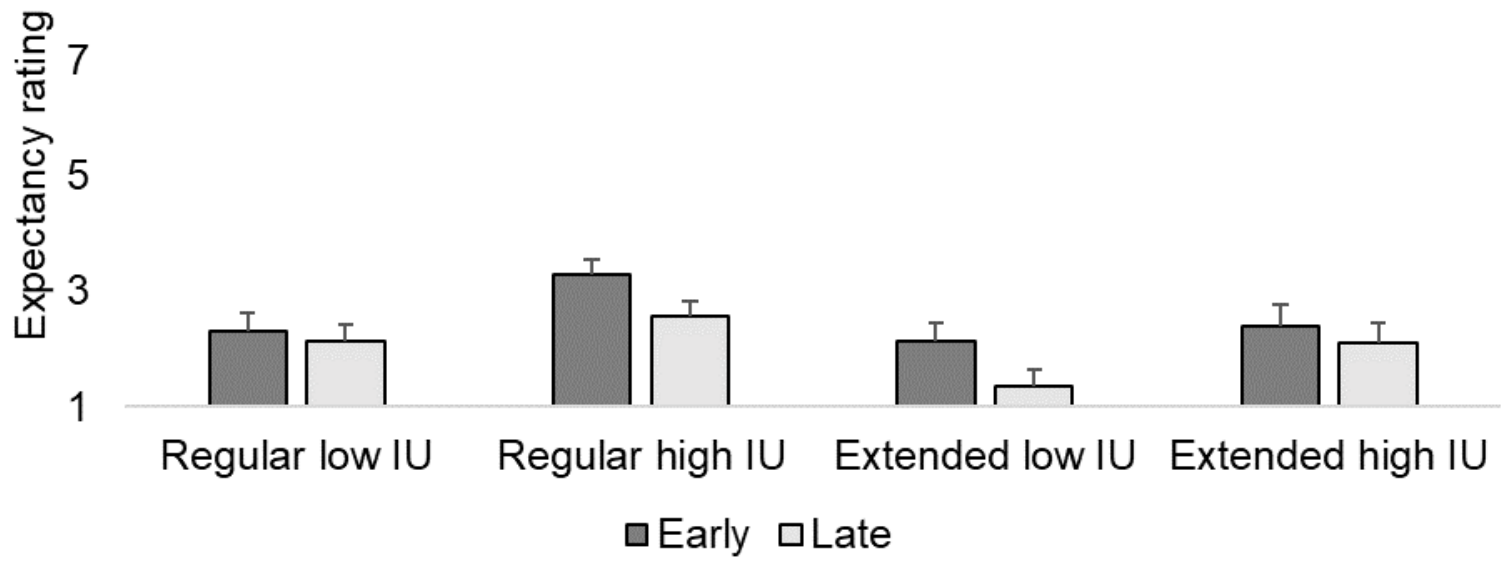




\section{Same-Day Extinction}

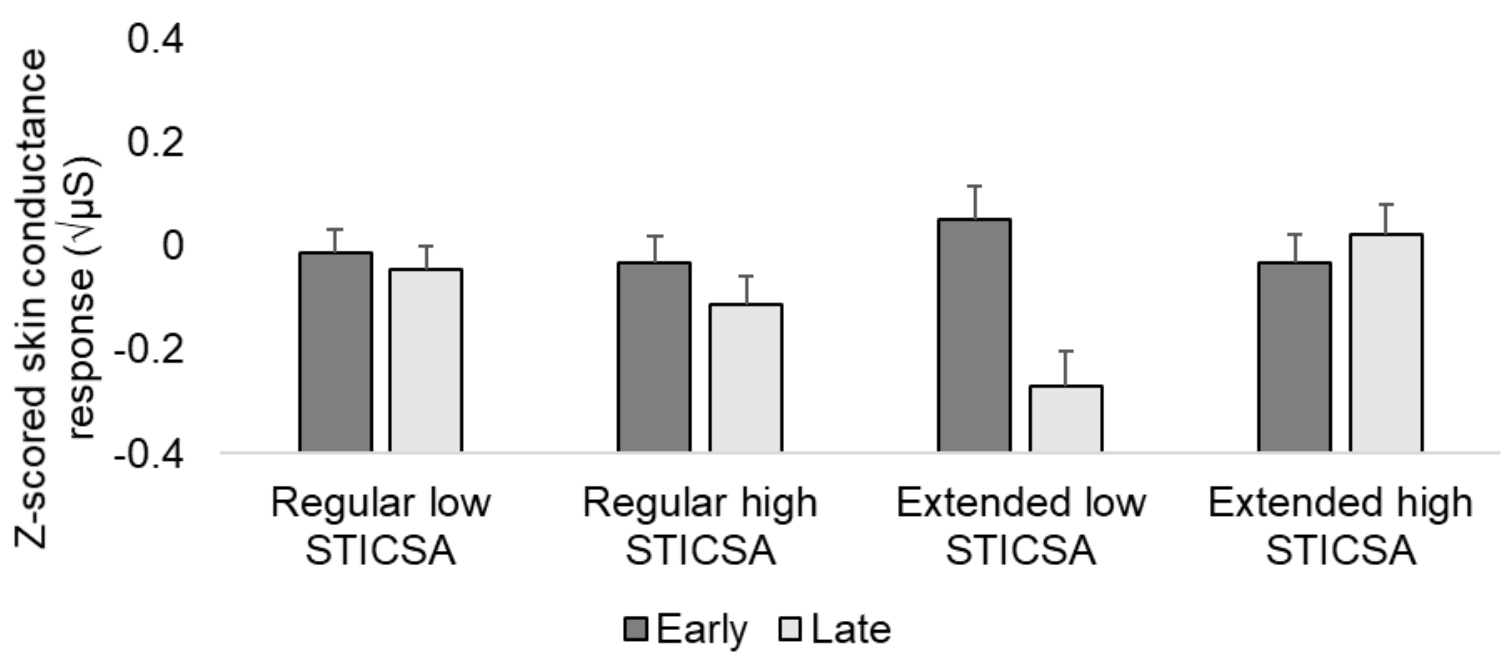

\section{Next-Day Extinction}

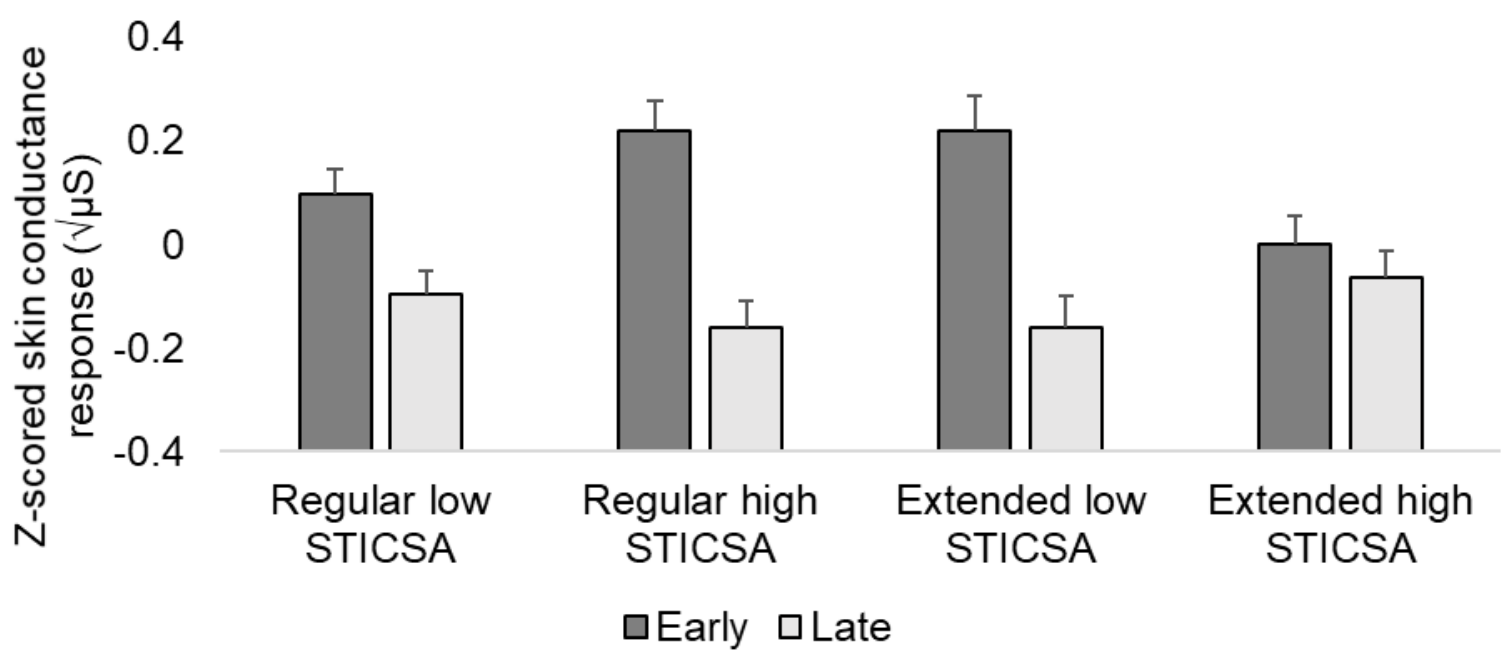




\section{Same-Day Extinction}
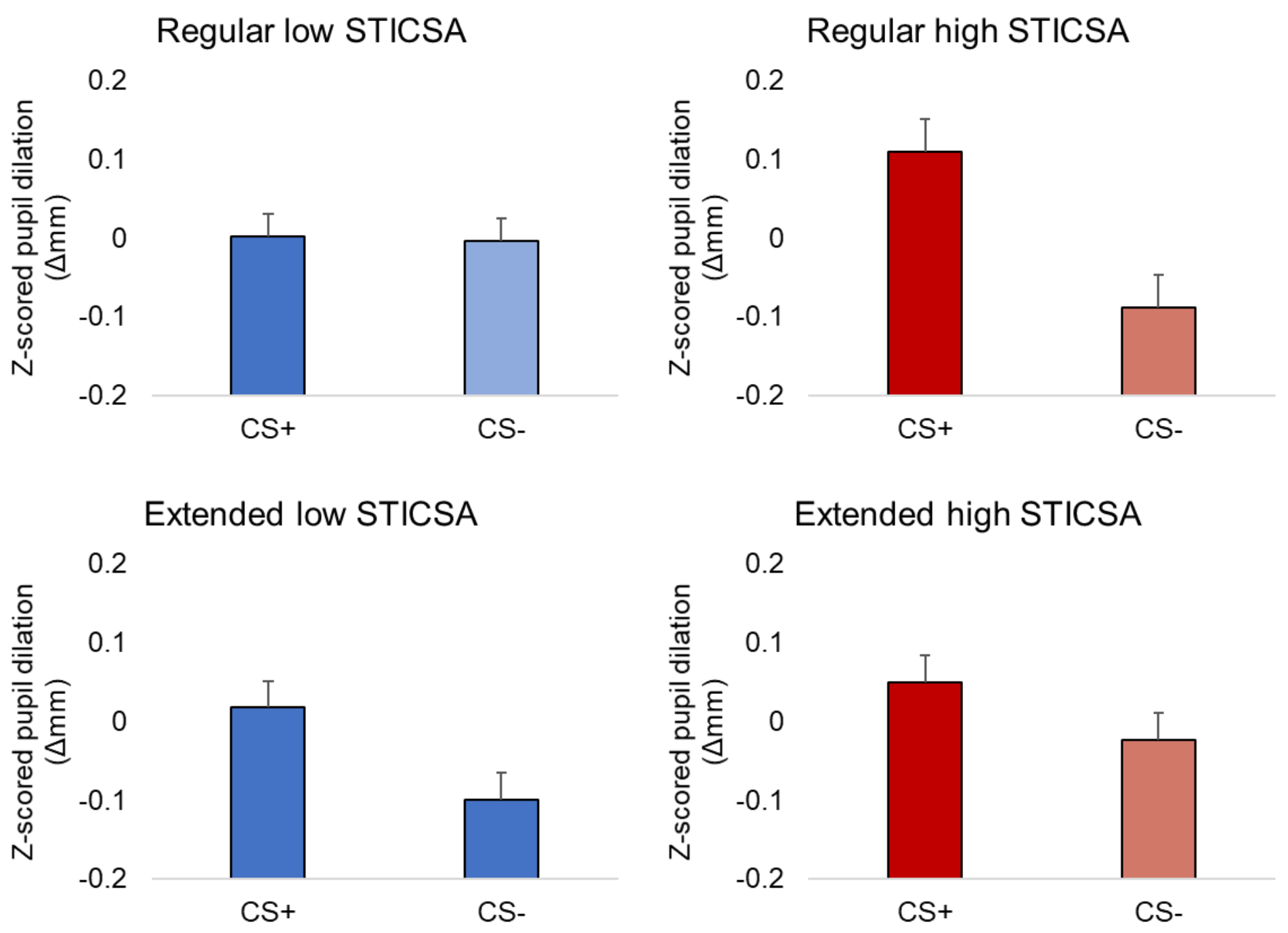


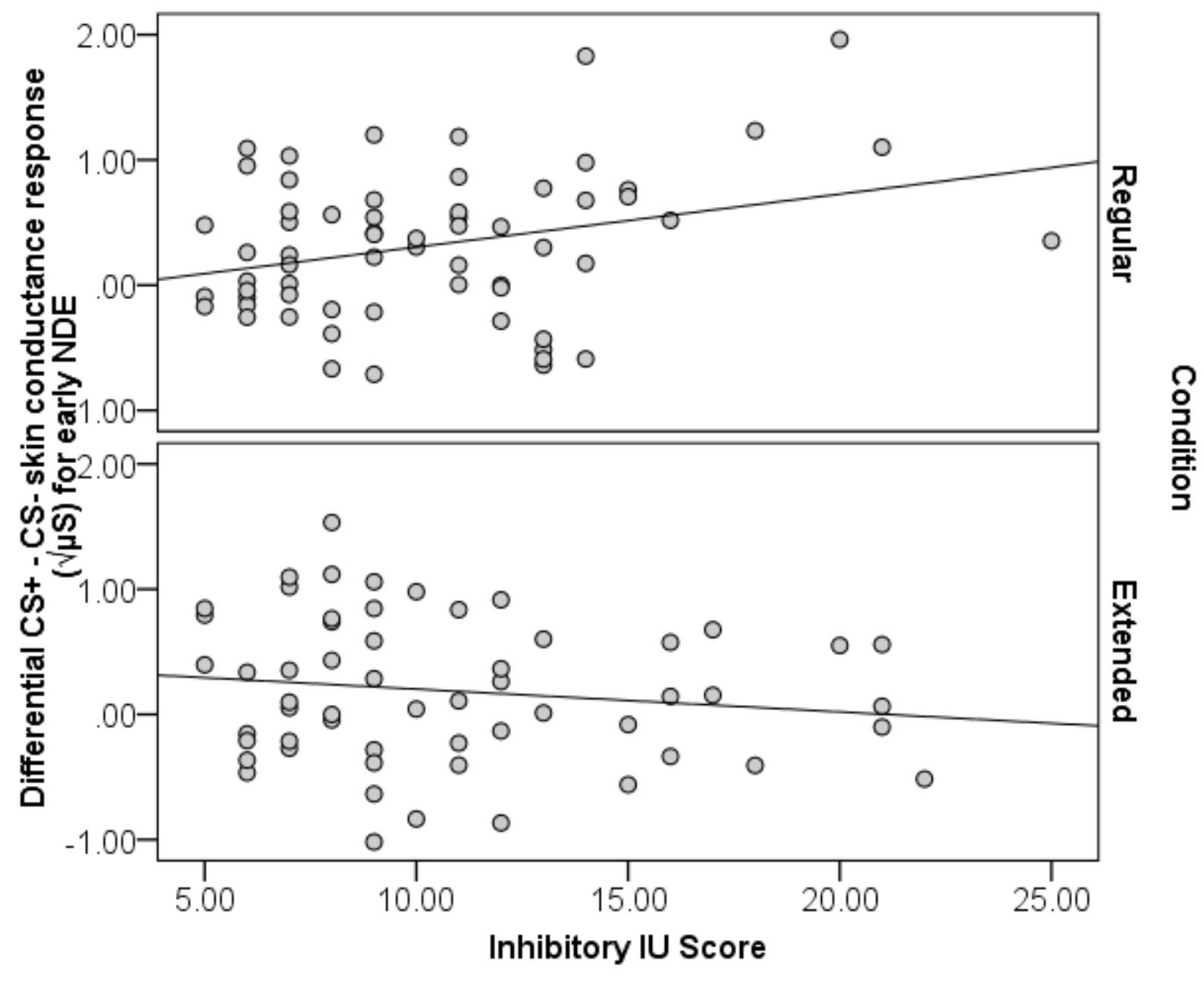

